\title{
Biliary lipid composition in idiopathic bile acid malabsorption
}

\author{
M Fracchia, S Pellegrino, P Secreto, A Pera, G Galatola
}

\begin{abstract}
Background-Chronic diarrhoea is the clinical hallmark of patients presenting with idiopathic bile acid malabsorption. Its pathogenesis is unknown; colonic water secretion can be induced by dihydroxy bile acids, but it is not known whether enrichment of the bile acid pool with these bile acids occurs in such patients. Furthermore, bile acid malabsorption is known to affect biliary lipid composition, but no information is available for the idiopathic type.

Aims-To verify: $(a)$ whether diarrhoea in patients with idiopathic bile acid malabsorption is associated with enrichment of the bile acid pool with dihydroxy bile acids; and (b) whether supersaturation with cholesterol of duodenal bile occurs in such patients as a result of chronic bile acid depletion.
\end{abstract}

Patients-Thirteen patients with idiopathic bile acid malabsorption diagnosed according to abnormal ${ }^{75}$ SeHCAT test and absence of other organic diseases, and 23 control subjects.

Methods-Bile rich duodenal fluid was collected during intravenous ceruletide infusion in the fasting state. Biliary lipids were analysed by enzymatic assays and bile acids by high performance liquid chromatography.

Results-Patients with idiopathic bile acid malabsorption had a cholesterol saturation index similar to controls. Bile acid composition showed only a decrease in percentage cholic acid (29 (2)\% versus 36 (2)\%; p<0.05); the dihydroxy:trihydroxy bile acid ratio was similar to controls.

Conclusions-Patients with idiopathic bile acid malabsorption do not have an increased risk of forming cholesterol gallstones. The mechanism of diarrhoea does not seem to depend on an enrichment of the bile acid pool with dihydroxy bile acids.

(Gut 1998;43:812-816)

Keywords: primary bile acid malabsorption; bile acids; diarrhoea; ${ }^{75} \mathrm{SeHCAT}$; biliary lipids; cholesterol saturation index Umberto I, Torino, Italy

S Pellegrino

Correspondence to: Dr G Galatola,

Gastroenterology Unit,

Ospedale Mauriziano

Umberto I, Largo Turati, 62

I-10128 Torino, Italy.

Accepted for publication 4 June 1998

Idiopathic, or type II, bile acid malabsorption (IBAM) is a condition characterised by chronic or intermittent diarrhoea in the presence of bile acid malabsorption of unknown origin and a symptomatic response to cholestyramine. Since its first description in 1973, it has been considered a rare condition. ${ }^{1}$ However, it is possible that the condition has been underdiagnosed, as its identification has for many years relied on showing excess faecal losses of bile acids, which requires the unpleasant handling of faeces and technical equipment not universally available. The availability of ${ }^{75} \mathrm{SeHCAT}$ $\left({ }^{75}\right.$ selena homocholic acid taurine) as a test substance for measuring bile acid turnover rate using abdominal scintigraphy has provided a much simpler procedure for diagnosing bile acid malabsorption. ${ }^{2-4}$ Consequently, IBAM has progressively seemed a more frequent condition than previously appreciated. ${ }^{56} \mathrm{~A}$ multicentre study has recently estimated that its prevalence is over $50 \%$ in patients with functional diarrhoea and even higher in those with an increased faecal wet weight.

The pathogenesis of IBAM is still unknown, and different mechanisms have been suggested. Morphological changes in terminal ileum biopsy specimens have been described in three patients with abnormal immune function, and an autoimmune disorder has been hypothesised as a possible mechanism leading to IBAM,${ }^{8}$ but these findings have not been confirmed. A defect in ileal bile acid uptake measured ex vivo has been suggested in two young patients with IBAM. ${ }^{9}$ Using more sophisticated techniques, Van Tilburg et al have not been able to confirm a bile acid carrier defect in biopsy specimens of the terminal ileum. ${ }^{10}$ Finally, mutations in the ileal sodium dependent bile acid transporter gene have been reported in members of a family with congenital idiopathic bile acid malabsorption. ${ }^{11}$ In the absence of a well defined disorder in the active bile acid absorption system, other hypotheses have been suggested, such as increased small intestinal motility and increased total bile acid pool, to an extent to overcome the ileal active absorption efficiency. ${ }^{12}$

A similar uncertainty exists over the possible mechanisms causing diarrhoea in patients with IBAM. Dihydroxy bile acids, such as chenodeoxycholic and deoxycholic acid, are known to inhibit colonic sodium reabsorption, thus causing diarrhoea. Although enrichment of the bile acid pool with dihydroxy bile acids has been hypothesised as possibly contributing to diarrhoea in IBAM, ${ }^{6}{ }^{10}$ there are no data on the composition of the bile acid pool in such patients.
Chronic bile acid malabsorption is known to alter biliary lipid and bile acid composition. Information is available for type I or organic bile acid malabsorption, ${ }^{13}{ }^{14}$ but not for patients with IBAM. We only found one report of two young patients with IBAM in a study of five individuals with bile acid malabsorption, in 
Table 1 General characteristics of the patients with idiopathic bile acid malabsorption (IBAM)

\begin{tabular}{lllllll}
\hline $\begin{array}{l}\text { Patient } \\
\text { number }\end{array}$ & Sex & $\begin{array}{l}\text { Age } \\
\text { (years) }\end{array}$ & BMI & $\begin{array}{l}\text { Faecal weight } \\
\text { (g/day) }\end{array}$ & Stools/week & $\begin{array}{l}{ }^{75} \text { SeHCAT } t^{\prime / 2} \\
\text { (days) }\end{array}$ \\
\hline 1 & $\mathrm{~F}$ & 56 & 20.1 & 250 & 25 & 1.58 \\
2 & $\mathrm{~F}$ & 32 & 18.0 & 333 & 35 & 0.01 \\
3 & $\mathrm{~F}$ & 25 & 17.5 & 180 & 34 & 1.00 \\
4 & $\mathrm{~F}$ & 30 & 18.4 & 210 & 30 & 0.97 \\
5 & $\mathrm{M}$ & 38 & 24.6 & 220 & 25 & 1.34 \\
6 & $\mathrm{~F}$ & 41 & 25.6 & 150 & 26 & 1.43 \\
7 & $\mathrm{M}$ & 34 & 23.1 & 210 & 22 & 1.62 \\
8 & $\mathrm{~F}$ & 43 & 17.8 & 210 & 24 & 1.74 \\
9 & $\mathrm{~F}$ & 58 & 26.0 & 260 & 24 & 0.88 \\
10 & $\mathrm{M}$ & 28 & 21.2 & 220 & 23 & 1.50 \\
11 & $\mathrm{M}$ & 65 & 23.0 & 250 & 22 & 1.14 \\
12 & $\mathrm{M}$ & 39 & 19.5 & 250 & 24 & 0.14 \\
13 & $\mathrm{~F}$ & 39 & 25.4 & 230 & 23 & 0.74 \\
Mean & $\mathrm{F} / 5 \mathrm{M}$ & 40.7 & 21.5 & 228 & 26 & 1.08 \\
SEM & - & 3.0 & 0.9 & 12 & 1 & 0.14 \\
\hline
\end{tabular}

BMI, body mass index.
EXPERIMENTAL PROCEDURE

Informed verbal consent for obtaining bile samples was obtained from all subjects and controls according to a protocol that was approved by the local ethical committee.

Duodenal bile was collected from all patients with IBAM and from 23 healthy subjects with normal bowel habit who served as controls (mean age 39 years; 12 men and 11 women). The procedure was carried out in the morning after overnight fasting. A nasoduodenal tube was positioned into the third portion of the duodenum under fluoroscopic guidance. A $5 \mathrm{ml}$ aliquot of bile rich duodenal fluid was then collected during intravenous infusion of 50 ng/kg ceruletide (Takus, Farmitalia, Milan, Italy).

The concentration of total bile acid, ${ }^{17}$ whom biliary lipids were measured before and after puberty. The authors found that cholesterol supersaturation developed in duodenal bile after puberty. ${ }^{15}$

The aim of our study was to measure biliary lipid and bile acid composition of patients with IBAM, in order to assess: (a) whether their diarrhoea is associated with biliary enrichment with dihydroxy bile acids; and (b) whether supersaturation with cholesterol of duodenal bile occurs as a consequence of chronic bile acid depletion, indicating an increased risk of cholesterol gall stone formation.

\section{Patients and methods}

We studied 13 patients with IBAM (mean age 40 years; five men and eight women). All patients were referred to our hospital because of a history of chronic/intermittent diarrhoea of more than six months duration. Diarrhoea was defined as an abnormal increase in bowel motions (more than 20 per week) and/or a faecal wet weight more than $600 \mathrm{~g} / 72$ hours. No patient reported a history of food intolerance, use of drugs possibly related to diarrhoea, including laxatives, or alcohol abuse. No patient had a history of major abdominal surgery. Routine blood chemistry, thyroid hormones, and tests for assessing nutritional status were in the normal range. Repeated search for bacterial or parasitic infections in the stools was negative.

All patients had a normal abdominal and intestinal ultrasonography and normal upper gastrointestinal endoscopy with normal morphology of duodenal biopsy specimens. Small bowel enteroclysis and colonoscopy with multiple biopsies (with inspection and biopsies of the last $10 \mathrm{~cm}$ of the terminal ileum when possible) were negative.

After the exclusion of known causes of diarrhoea, patients were submitted to a ${ }^{75} \mathrm{SeHCAT}$ test. The ${ }^{75} \mathrm{SeHCAT}$ test was performed as previously described, ${ }^{3}{ }^{16}$ assessing the exponential decrease of radioactivity over the gall bladder area by collimated gammacamera for seven consecutive days. ${ }^{3}$ Bile acid malabsorption was diagnosed when ${ }^{75} \mathrm{SeHCAT}$ half life was less than two days. ${ }^{16}$ cholesterol, ${ }^{18}$ and phospholipid ${ }^{19}$ was measured enzymatically. All bile samples satisfied the requirement of a total lipid concentration greater than $5 \mathrm{~g} / \mathrm{dl}$, thus rendering possible a reliable calculation of cholesterol saturation index (CSI). ${ }^{20} \mathrm{CSI}$ was calculated using the polynomial equation of Thomas and Hofmann ${ }^{21}$ based on cholesterol solubility lines described by Hegardt and Dam. ${ }^{22}$

Analysis of the conjugated bile acids (CA, cholic acid; CDCA, chenodeoxycholic acid; DCA, deoxycholic acid; UDCA, ursodeoxycholic acid; LCA, lithocholic acid) was carried out by high performance liquid chromatography (HPLC) using a modification of a previously described technique. ${ }^{23}$ Analytical grade reagents and deionised distilled water were used. Conjugated bile acids and phenol (internal standard) were purchased from Sigma Co. (St Louis, Missouri, USA). Aqueous $\mathrm{KH}_{2} \mathrm{PO}_{4}, 2.0 \mathrm{~g} / 1$ (A), $\mathrm{H}_{3} \mathrm{PO}_{4} 85 \%, 2.0 \mathrm{ml} / 1$ (B) solutions, and gradient grade acetonitrile (C) (Merck, Darmstadt, Germany) were used as the mobile phase. Bile $(200 \mu \mathrm{l})$ was diluted to $4.0 \mathrm{ml}$ with $100 \%$ ethanol, brought to boiling point for 5-10 minutes, and left overnight at room temperature in the dark. The mixture was spiked with $100 \mu \mathrm{l}$ ethanolic phenol solution $(0.5 \mathrm{mg} / \mathrm{ml})$, shaken well, and filtered through a $0.22 \mu \mathrm{m}$ Millipore filter; $4.0 \mathrm{ml}$ of an ethanolic solution containing $1.0 \mathrm{mg} / \mathrm{ml}$ of each bile acid, spiked with $100 \mu \mathrm{l}$ of internal standard, was used as the standard. A $20 \mu \mathrm{l}$ aliquot of sample or standard was injected into the chromatograph.

A Merck Hitachi (Merck, Darmstadt, Germany) liquid chromatograph (L-6200A Intelligent Pump) equipped with a UV-VIS variable wavelength detector (L-4250 UV-VIS detector), an automatic injection autosampler (AS2000A Autosampler), and a column oven (L-5025 Column Thermostat) were used. An octadecylsylil LiChroCART $250 \times 4 \mathrm{~mm}$ HPLC-Cartridge Superspher $100 \mathrm{RP}-184 \mu \mathrm{m}$ column (Merck) was used throughout with a LiChroCART $4 \times 4 \mathrm{~mm}$ HPLC-Cartridge LiChrospher $100 \mathrm{RP}-185 \mu \mathrm{m}$ (Merck) guard column. Elution was performed at $1.0 \mathrm{ml} / \mathrm{min}$ flow rate at $25^{\circ} \mathrm{C}$. A multistep linear gradient, starting at time 0 with A:B:C eluent composition at 41:31:28 ( $\mathrm{vol} / \mathrm{vol} \%)$ proportion, was imposed over 90 minutes. The initial composi- 
Table 2 Biliary lipid ratios in control subjects and patients with idiopathic bile acid malabsorption (IBAM)

\begin{tabular}{llll}
\hline Group & BA:CHOL & BA:PL & PL:CHOL \\
\hline Controls & $19.8(4.1)$ & $4.3(0.5)$ & $4.5(0.6)$ \\
IBAM & $26.1(7.2)$ & $5.7(0.4)$ & $4.2(0.9)$ \\
p Value & NS & NS & NS \\
\hline
\end{tabular}

Results expressed as mean (SEM).

BA, bile acids; CHOL, cholesterol; PL, phospholipids.

tion, maintained for 10 minutes, was brought to $38: 29: 33$ within five minutes; this was maintained for the following 10 minutes. The composition was then linearly brought to $2: 2: 96$ during the following 45 minutes and then to the original conditions within two minutes. The column was reequilibrated for 18 minutes before the next injection. The acetonitrile gradient effectively served to elute more retained contaminants, which otherwise could appear during subsequent chromatograms. Optimum chromatographic performance was obtained by accurate preparation of the mobile phase. The column was periodically cleaned by flushing with pure acetonitrile. Peak height was

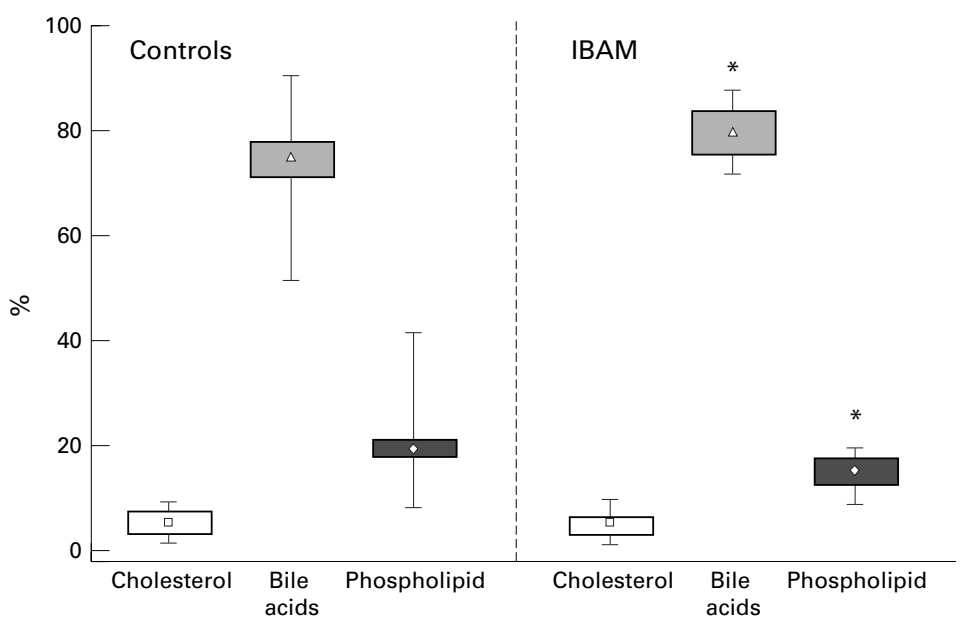

Figure 1 Data distribution for percentage cholesterol, bile acids, and phospholipid in controls and patients with IBAM. Symbols indicate median values, boxes indicate 75 th and 25 th percentiles, and whiskers indicate minimum and maximum values of the range. ${ }^{*} p<0.05$ versus controls.

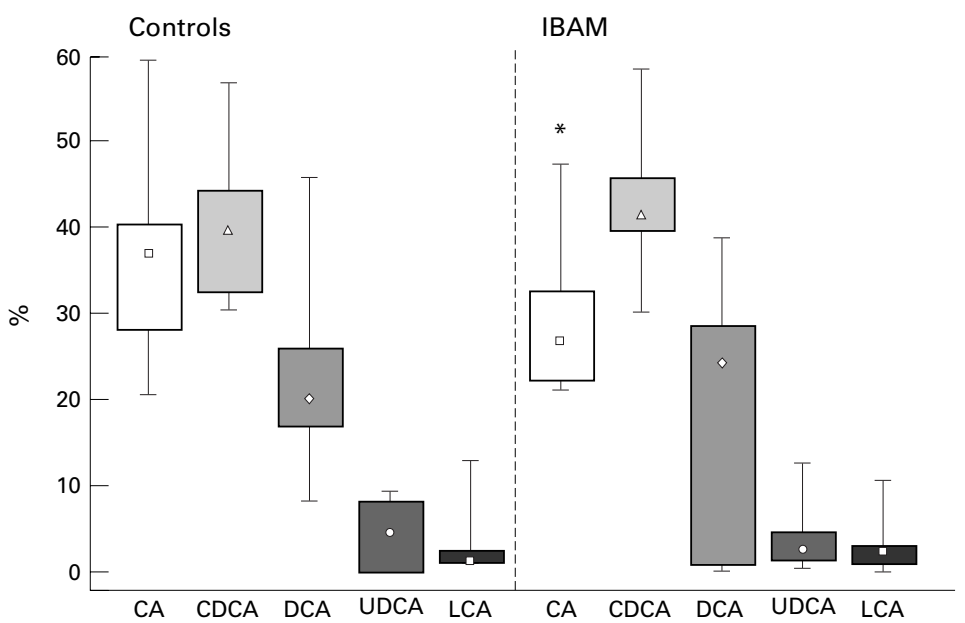

Figure 2 Data distribution for percentage bile acid composition (CA, cholic acid;CDCA, chenodeoxycholic acid; DCA, deoxycholic acid; UDCA, ursodeoxycholic acid; LCA, lithocholic acid) in controls and patients with IBAM. Symbols indicate median values, boxes indicate 75 th and 25 th percentiles, and whiskers indicate minimum and maximum values of the range. ${ }^{\star} p<0.05$ versus controls. measured using a Merck Hitachi D-2500 Chromato-Integrator. The detection was performed at $200 \mathrm{~nm}$, the detector output was set at 0.002 absorbance units at full scale, and the integrator input was $128-256 \mathrm{mV}$ at full scale. The reproducibility, assessed by repeated assays of bile samples, and the accuracy, evaluated by adding increasing concentrations of standards to bile acid specimens, were always greater than $98 \%$.

STATISTICAL ANALYSIS

Results were expressed as mean (SEM). Data distribution was shown by plotting median values, 75 th and 25 th percentiles, maximum and minimum values. Significant differences were assessed using the Mann-Whitney U test. Linear regression analysis was used for assessing the existence of significant correlations. Values of $\mathrm{p}$ less than 0.05 were considered significant.

\section{Results}

Table 1 shows the general characteristics of the patients. All patients with IBAM had a normal body mass index. Diarrhoea was present in all patients with IBAM, as defined by increased faecal wet weight and/or weekly stool frequency. All patients responded to cholestyramine administration-begun after completion of the ${ }^{75} \mathrm{SeHCAT}$ test and bile collection-by a decrease in stool frequency (no more than 15 per week) and disappearance of symptoms (abdominal pain, bloating, faecal urgency).

Cholesterol saturation index was $0.97(0.12)$ in patients with IBAM versus $1.02(0.09)$ in controls (NS). Figure 1 shows the distribution of data for biliary lipid composition. Patients with IBAM had significantly higher percentage bile acid values and lower percentage phospholipid values. Mean (SEM) values were 5.0 $(0.7) \%$ for percentage cholesterol in patients with IBAM versus 5.8 (0.6)\% in controls (NS); $80.2(1.3) \%$ versus $73.5(2.0) \%(\mathrm{p}<0.05)$ for total bile acids; and $14.8(0.9) \%$ versus 20.6 $(1.7) \%(\mathrm{p}<0.05)$ for phospholipids. Table 2 shows that no significant differences for biliary lipid ratios were found between patients and healthy subjects.

Figure 2 shows the distribution of data for biliary bile acid composition. A significant depletion of cholic acid was observed in patients with IBAM. No difference was observed for the other bile acids, although percentage deoxycholic acid showed a wide variability in patients with IBAM, ranging from $0.13 \%$ to $38.67 \%$; three patients had less than $1 \%$ deoxycholic acid in their bile. Mean (SEM) values were $29(2) \%$ in patients with IBAM versus $36(2) \%$ in controls $(\mathrm{p}<0.05)$ for $\mathrm{CA} ; 44$ (2) $\%$ versus 40 (2) $\%$ (NS) for CDCA; 20 (4)\% versus 22 (2)\% (NS) for DCA; 4 (1)\% versus 5 (2) $\%$ (NS) for UDCA; and 3 (1)\% versus 4 (2) $\%$ (NS) for LCA.

No difference was observed for dihydroxy: trihydroxy bile acid ratio between patients with IBAM and controls (2.12(0.19) versus 1.86 (0.16); NS). 
${ }^{75} \mathrm{SeHCAT}$ half life was not associated with the percentage of cholic and deoxycholic acid, nor with cholesterol saturation index.

\section{Discussion}

We have shown that patients with idiopathic bile acid malabsorption have a cholesterol saturation index similar to healthy subjects, indicating that they do not have an increased risk of forming cholesterol gall stones. Data on cholesterol saturation of duodenal bile in patients with bile acid malabsorption of organic cause are conflicting. Earlier studies reported that duodenal bile becomes supersaturated with cholesterol following ileal resection or disease..$^{14}$ Later, Färkkilä et al however, found a normal cholesterol saturation of duodenal bile in 29 patients after ileal resection. ${ }^{24}$ Similar results were obtained from Einarsson's group in patients with Crohn's disease following ileal resection. ${ }^{25}$ Similarly, Koivisto and Miettilnen found cholesterol saturation of duodenal bile to be normal in patients with partial ileal bypass ${ }^{26}$ and we found this to be true also in patients with ileoanal anastomosis and total colectomy, ${ }^{27}$ who have bile acid malabsorption according to the ${ }^{75} \mathrm{SeHCAT}$ test. ${ }^{16}$

The biliary lipid composition of our patients with IBAM showed a percentage enrichment in bile acid, with a corresponding decrease in percentage phospholipid. These findings are similar to those reported by Färkkilä et al in ileal resection patients, although the notable decrease in percentage phospholipids did not reach statistical significance in this study. ${ }^{24} \mathrm{~A}$ possible explanation of this pattern may be an increase in faecal losses of bile acids, causing increased hepatic cholesterol synthesis, which in turn is mainly diverted to bile acid synthesis. ${ }^{24}$ Despite the altered percentage biliary lipids composition, biliary lipid ratios showed no differences between patients and controls, confirming that idiopathic bile acid malabsorption does not predispose to cholesterol gall stone formation. This is supported by a long term study in a cohort of 23 patients with IBAM, where cholelithiasis was not mentioned as a complication occurring in the natural history of this disorder. ${ }^{28}$

Bile acid composition in patients with IBAM was characterised by a significant decrease of cholic acid, without alteration in the dihydroxy to trihydroxy bile acid ratio. Thus, enrichment of the bile acid pool with dihydroxy bile acids does not seem to be the cause of diarrhoea in such patients. Theoretically, measurement of dihydroxy bile acid concentration in the aqueous phase of faeces could provide more information in this respect. As it is however generally observed, not all patients with IBAM pass liquid faeces unless IBAM is severe; their stools are mostly soft, making the measurement of bile acid concentration in the aqueous phase of centrifugate poorly representative of the actual concentration within the colon. Furthermore, faecal bile acid concentration can be affected by poorly controllable factors and shows large intraindividual and day to day variation, as observed by Setchell et $a .^{29}$ Finally, the composition of biliary bile acids more closely reflects the colonic exposure to bile acids. $^{30}$

The reduction in percentage cholic acid observed in our patients has also been reported in patients with bile acid malabsorption following partial ileal bypass ${ }^{31}$ : in these patients there was also an increase in the hepatic synthesis of both cholic and chenodeoxycholic acid to the same extent. A preferential reduction in the ileal absorption efficiency of cholic acid ${ }^{32}$ has been hypothesised to explain these results: we therefore suggest that the primary pathogenic mechanism of IBAM is a selective defect in bile acid absorption of the ileum. Definite demonstration of such a mechanism is however not available. However, recently Oelkers et al have described a family with congenital idiopathic bile acid malabsorption, where mutation in the ileal sodium dependent bile acid transporter gene has been identified. ${ }^{11}$ Whether our adult patients represent a more common subset of individuals with partial abnormalities in this gene, is not known, but this may be an attractive working hypothesis.

The mean percentage deoxycholic acid showed no differences between our patients and controls. Howewer, three patients had only traces of deoxycholic acid in their duodenal bile. A wide variability in percentage biliary deoxycholic acid but with an overall reduction by comparison with controls has been reported in the ileal resection patients of Lapidus and Einarsson's study, ${ }^{25}$ whereas Kern et al have reported deoxycholic acid concentrations ranging from a complete absence up to an increased percentage in their ileal resection patients. ${ }^{33}$ Biliary concentrations of deoxycholic acid were found not to correlate with the length of ileal resection. ${ }^{34}$ Accordingly, no correlation existed in our patients with IBAM between ${ }^{75} \mathrm{SeHCAT}$ half life and percent deoxycholic acid. This is in line with the hypothesis that deoxycholic acid concentrations are not dependent on the severity of faecal bile acid loss. ${ }^{33}$ A role of abnormal intestinal bacteria has been suggested to explain these findings. ${ }^{25}{ }^{33}$ There is growing evidence that enzymes produced by the colonic bacteria and the degree of slowing of colonic transit time are of paramount importance in determining the level of production of deoxycholic acid, ${ }^{35}$ which seems to be strongly associated with cholesterol supersaturation of bile, and therefore formation of cholesterol gall stones. ${ }^{37}$ The absence of an enrichment of the bile acid pool with deoxycholic acid further supports the hypothesis that patients with IBAM are not at increased risk of forming cholesterol gall stones.

In conclusion, we have shown that patients with IBAM are not at increased risk of forming cholesterol gall stones despite evidence of increased bile acid loss from their enterohepatic circulation. The mechanism of the resulting diarrhoea does not seem to depend on an enrichment of the bile acid pool with deoxycholic acid or other dihydroxy bile acids that might stimulate water secretion from the colonic mucosa. 
1 Hess Thaysen E, Pedersen L. Diarrhoea associated with idiopathic bile acid malabsorption: fact or fantasy? Danish Med Bull $1973,20.174$

Nyhlin H, Merrick MV, Eastwood MA, et al. Evaluation of ileal function using 23-selena-25-homotaurocholate, a $\gamma$-labeled conjugated bile acid. Initial clinical assessment. Gastroenterology 1983;84:63-8.

3 Ferraris R, Jazrawi RP, Bridges C, et al. Use of a gamma labelled bile acid (75-SeHCAT) as a test of ileal function. Methods of improving accuracy. Gastroenterology 1986;90 1129-36.

4 Galatola G, Jazrawi RP, Bridges C, et al. Direct measurement of first-pass ileal clearance of a bile acid in humans. Gastroenterology 1991;100:1100-5.

5 Merrick MV, Eastwood MA, Ford MJ. Is bile acid malabsorption underdiagnosed? An evaluation of accuracy of diagnosis by measurement of SeHCAT retention. BMF 1985;290:665-8.

6 Williams AJK, Merrick MV, Eastwood MA. Idiopathic bile acid malabsorption-a review of clinical presentation, acid malabsorption - a review of clinical presentation,

diagnosis, and response to treatment. Gut 1991;32:1004-6. Galatola G and the Italian 75SeHCAT Multicenter Study
Group. The prevalence of bile acid malabsorption in irritable bowel syndrome and the effect of cholestyramine: an uncontrolled open multicenter study. Eur $\mathcal{F}$ Gastroenterol Hepatol 1992;4:533-7

8 Popovic OS, Kostic KM, Milovic VB, et al. Primary bile acid malabsorption. Gastroenterology 1987;92:1851-8.

9 Heubi JE, Balistreri WF, Fondacaro JD, et al. Primary bile acid malabsorption: defective in vitro ileal active bile acid transport. Gastroenterology 1982;83:804-11.

10 van Tilburg AJP, de Rooij FWM, van den Berg JWO, et al. Primary bile acid diarrhoea without an ileal carrier defect: quantification of active bile acid transport across the ileal brush border membrane. Gut 1991;32:500-3

11 Oelkers P, Kirby LC, Heubi JE, et al. Primary bile acid malabsorption caused by mutations in the ileal sodiumdependent bile acid transporter gene (SLC10A2). I Clin Invest 1997;99:1880-7.

12 van Tilburg AJP, de Rooij FWM, van den Berg JWO, et al. Primary bile acid malabsorption: a pathophysiologic and
clinical entity? Scand 7 Gastroenterol 1992;27(suppl 194): clinical

13 Dowling RH, Bell GD, White J. Lithogenic bile in patients with ileal dysfunction. Gut 1972;13:415-20.

14 Rutgeerts P, Ghoos Y, Vantrappen G. Effect of partial ileocolectomy and Crohn's disease on biliary lipid secretion. Dig Dis Sci 1987;32:1231-8.

15 Heubi JE, O'Connell NC, Setchell KDR. Ileal resection/ dysfunction in childhood predisposes to lithogenic bile only after puberty. Gastroenterology 1992;103:636-40.

16 Ferraris R, Galatola G, Barlotta A, et al. Measurement of bile acid half-life using 75SeHCAT in health and intestinal diseases. Comparison with $75 \mathrm{SeHCAT}$ abdominal retention methods. Dig Dis Sci 1992;37:225-32.

17 Talalay P. Enzymatic analysis of steroid hormones. Methods Biochem Anal 1960;8:119-43.

18 Roda A, Festi D, Sama C, et al. Enzymatic determination of cholesterol in bile. Clin Chim Acta 1975;64:337-41.

19 Qureshi NY, Murphy GM, Dowling RH. The enzymatic determination of total phospholipid in bile and bile-rich duodenal aspirates. Clin Chim Acta 1980;105:407-10.
20 Strasberg SM, Harvey PRC, Hofmann AF. Bile sampling, processing and analysis in clinical studies. Hepatology 1990; 12:176S-82S

21 Thomas PJ, Hofmann AF. A simple calculation of the lithogenic index of bile expressing biliary lipid composition on rectangular coordinates. Gastroenterology 1973;65:698700 .

22 Hegardt FG, Dam H. The solubility of cholesterol in aqueous solutions of bile salts and lecithin. Z Ernahrungswiss 1971;10:223-33.

23 Nakayama F, Nakagaki M. Quantitative determination of bile acids in bile with reverse-phase high performance liquid chromatography. F Chromatogr 1980;183:287-93.

24 Färkkilä MA. Biliary cholesterol and lithogeneity of bile in patients after ileal resection. Surgery 1988;104:18-25.

25 Lapidus A, Einarsson K. Effects of ileal resection on biliary lipids and bile acid composition in patients with Crohn's disease. Gut 1991;32:1488-91.

26 Koivisto P, Miettilnen TA. Gallbladder disease, bile composition and bile acid absorption after ileal bypass. Hepatogastroenterology 1990;37(suppl 2):49-54.

27 Galatola G, Fracchia M, Jazrawi RP. Effect of colectomy with ileo-anal anastomosis on the biliary lipids. Eur $\mathcal{F}$ Clin Invest 1995;25:534-8.

28 Luman W, Williams AJK, Merrick MV, et al. Idiopathic bile acid malabsorption: long-term outcome. Eur f Gastroenterol Hepatol 1995;7:641-5.

29 Setchell KDR, Ives JA, Cahmore GC, et al. On the homogeneity of stools with respect to bile acid composition and normal day-to-day variations: a detailed qualitative and quantitative study using capillary column gas chromatography-mass spectrometry. Clin Chim Acta 1987; 162:257-75.

30 Bayerdörffer E, Mannes GA, Richter WO, et al. Increased serum deoxycholic acid levels in men with colorectal adenomas. Gastroenterology 1993;104:145-51.

31 Koivisto PVI. Fecal and biliary bile acid composition after partial ileal bypass operation. Digestion 1988;39:52-60.

32 Einarsson KA, Grundy SM, Hardison WGM. Enterohepatic circulation rates of cholic acid and chenodeoxycholic acid in man. Gut 1979;20:1078-82.

33 Kern F Jr. Disappearance of deoxycholic acid after ileal resection. Gastroenterology 1973;64:123-7.

34 Mallory A, Kern F Jr, Smith J, et al. Patterns of bile acids and microflora in the human small intestine. I. Bile acids. Gastroenterology 1973;64:26-33.

35 Berr F, Kullak-Ublick GA, Paumgartner G, et al. $7 \alpha-$ Dehydroxylating bacteria enhance deoxycholic acid input and cholesterol saturation of bile in patients with gallstones. Gastroenterology 1996;111:1611-20.

36 Hussaini SH, Murphy GM, Kennedy C, et al. The role of bile composition and physical chemistry in the pathogenesis of octreotide-associated gallbladder stones. Gastroenterology 1994;107:1503-13.

37 Marcus SN, Heaton KW. Intestinal transit, deoxycholic acid and the cholesterol saturation of bile: three interrelated factors. Gut 1986;27:550-8. 